\title{
Perfect 2-coloring of the quartic graphs with order at most 8
}

\author{
Yazdan Golzadeh ${ }^{1} \cdot$ Mehdi Alaeiyan $^{2} \cdot$ Alireza Gilani $^{1}$
}

Received: 18 March 2019 / Accepted: 27 July 2019 / Published online: 5 September 2019

(c) The Author(s) 2019

\begin{abstract}
In this paper, we study perfect 2-coloring of the quartic graphs with at most 8 vertices. The problem of the existence of perfect coloring is a generalization of the concept of completely regular codes, given by Delsarte.
\end{abstract}

Keywords Perfect coloring $\cdot$ Equitable partition $\cdot$ Quartic graph $\cdot$ Perfect code $\cdot$ Regular graph

Mathematics Subject Classification $05 \mathrm{C} 15 \cdot 05 \mathrm{C} 78$

\section{Introduction}

Let $X=(V(X), E(X))$ be a connected graph. A vertex coloring with $m$ colors is perfect if there is a matrix $M=\left(\alpha_{i j}\right)_{i, j=1,2, \ldots m}$ such that the cardinal of vertices of label $j$ connected to a specified vertex of label $i$ is equal to $\alpha_{i, j}$.

In particular, all vertices of the same color in $X$ have the same degree. This type of coloring gives a partition of $V(X)$ that is called equitable partitions. These partitions of vertices have two properties as follows: The first property is that the vertices of each part $P_{i}$ induce a regular graph, and the second is the edges between $P_{i}$ and $P_{j}$ induce a half-regular graph. In Section 3, we find the parameter matrices of a perfect 2-coloring. Equitable partitions were previously studied in [7].

In fact, the concept of a perfect $m$-coloring is bridge of among algebraic combinatorics, graph theory and coding theory (including 1-perfect codes). For the definition of 1-perfect codes and more details, we refer the reader to [1].

This type of coloring is generalized the concept of completely regular codes introduced by P. Delsarte ([8]).The

Mehdi Alaeiyan

alaeiyan@iust.ac.ir

Yazdan Golzadeh

st_y_golzadeh@azad.ac.ir

Alireza Gilani

a_gilani@azad.ac.ir

1 Department of Mathematics, South Tehran Branch, Islamic Azad University, Tehran, Iran

2 Department of Mathematics, Iran University of Science and Technology, Narmak, 16844 Tehran, Iran problem for the Johnson graphs has been studied (see[4], see[3], see [6]).

In subsequent attempts, the problem of existence of perfect coloring was examined on other graphs. For example, Fon-Der-Flass settled perfect coloring with two colors of $n$-dimensional hypercube graphs $Q_{n}$ for $n<24$ (see [5]). In this paper, we will list all parameter matrices of perfect 2-coloring of the quartic graphs up to order 8 .

\section{Notations and preliminaries result on the existence of perfect coloring}

In this section, some basic definitions that will be used in this paper are given and we declare some conditions for existence of perfect 2-coloring of the quartic graphs.

In this paper, all the graphs are simple. A graph $X=(V(X), E(X))$ is $k$-regular if for every $v \in V(X)$ we have $\operatorname{deg}(v)=k$, where $k$ is a nonnegative integer number. In particular, graphs that are regular of degrees 3, 4, 5 and 6 are called, respectively, cubic, quartic, quantic and sixtic.

In the following remark, we classify the quartic graphs with at most 8 vertices (see [9]).

Remark 1 The quartic graphs are divided into four classes based on their vertices. This classification is shown in Figs. 1, 2, 3 and 4. For simplicity, we name the vertices as shown in these figures.

We now introduce the concept of perfect coloring. 


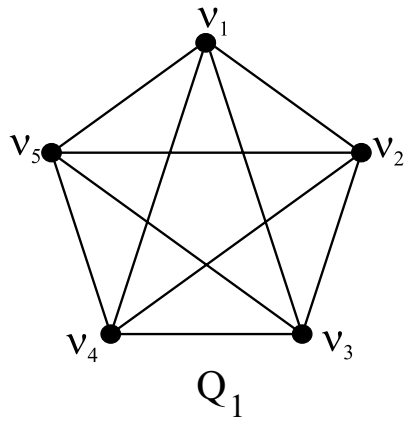

Fig. 1 Class 1: The quartic graph with 5 vertices

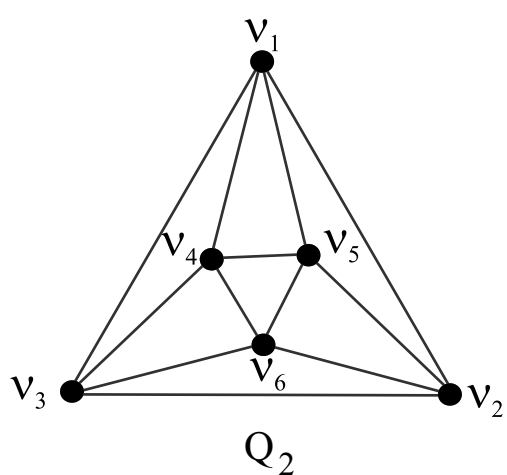

Fig. 2 Class 2: The quartic graph with 6 vertices

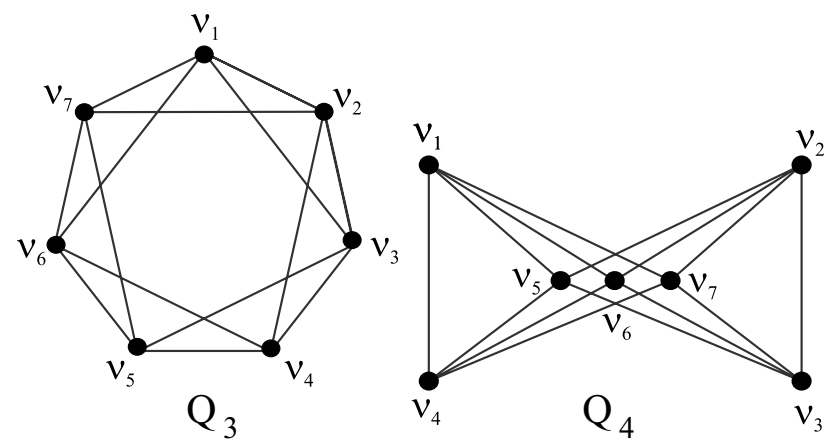

Fig. 3 Class 3: The quartic graph with 7 vertices

Definition 1 Let $X=(V(X), E(X))$ be a connected graph. A perfect $m$-coloring with parameter matrix $M=\left(\alpha_{i j}\right)_{i, j=1,2, \ldots m}$ is a map $C$ from $V(X)$ to the set of colors $\{1,2, \ldots, m\}$ such that $C$ is surjective, and for a fix vertices $v$ where $C(v)=i$ we have $\mid\{w \in V(X) \mid w$ is adjacent to $\mathrm{v}, C(w)=j\} \mid=\alpha_{i j}$, for all $i, j$.

In this case, we say $C$ is a P.m.C (or just P.C). Here, we study P.2.C, where two colors are red and blue.

In this case, we consider the parameter matrix of P.2.C generated by replacing the colors with the primary coloring is equal. This means the parameter matrix $\left(\begin{array}{cc}a & b \\ a^{\prime} & b^{\prime}\end{array}\right)$ is equal to the parameter matrix $\left(\begin{array}{cc}b^{\prime} & b \\ a^{\prime} & a\end{array}\right)$. We call equality of parameter matrix. Some properties of P.C have been studied recently (see $[4,6,8])$. The main result of them is the next proposition that enumerates the cardinal number of blue vertices in a $P .2 . C$ with matrix $M=\left(\alpha_{i j}\right)_{i, j=1,2}($ see [3]).

Proposition 1 Assume that $B$ is the set of all blue vertices in a P.2.C of a graph $X=(V(X), E(X))$ with matrix $\left(\begin{array}{cc}a & b \\ a^{\prime} & b^{\prime}\end{array}\right)$. Then we have

$|B|=\frac{a^{\prime}|V(X)|}{b+a^{\prime}}$.

Now, the following remark provides useful information about calculation P.2.C of the quartic graphs.

Remark 2 Suppose that $X=(V(X), E(X))$ be a simple connected graph. Then necessary conditions for existence of P.2.C of $X$ with parameter matrix $M=\left(\begin{array}{cc}a & b \\ a^{\prime} & b^{\prime}\end{array}\right)$ are listed as follows:

1. Degree condition: This simple condition that results from the regularity of $X$ tells us:

$a+b=a^{\prime}+b^{\prime}=4$.

2. Connectedness condition: Since the graph $X$ is connected, another condition is induced as follows:

$b, a^{\prime} \neq 0$.

We will define a new definition for the third condition. The number $\mu$ is called an eigenvalue of a graph $X$, if $\mu$ is an eigenvalue of the adjacency matrix of $X$. The number $\mu$ is called an eigenvalue of a $P .2 . C$ with the matrix $M$, if $\mu$ is an eigenvalue of $M$.

The next theorem gives us the third condition and the connection between two types of eigenvalues (see [4]):

Theorem 1 Let $C$ be a P.m.C of a graph X. Thus any eigenvalues of $C$ are an eigenvalues of $X$.

It has been proved that a P.2.C of a k-regular graph X has just two eigenvalues (see [4]).

Proposition 2 If $C$ be a P.2.C with parameters matrix $M=\left(\alpha_{i, j}\right)_{i, j=1,2}$ of a regular graph $X$ of valency $k$, then the numbers $\alpha_{11}-\alpha_{21}$ and $k$ are eigenvalues of $C$ and therefore are eigenvalues of $X$.

Lemma 1 Given an arbitrarily connected quartic graph X, the following ten matrices are the only matrices that can be selected as acceptable parameter matrices of a perfect 2-coloring $C$ of $X$ : 
Fig. 4 Class 4: The quartic graph with 8 vertices
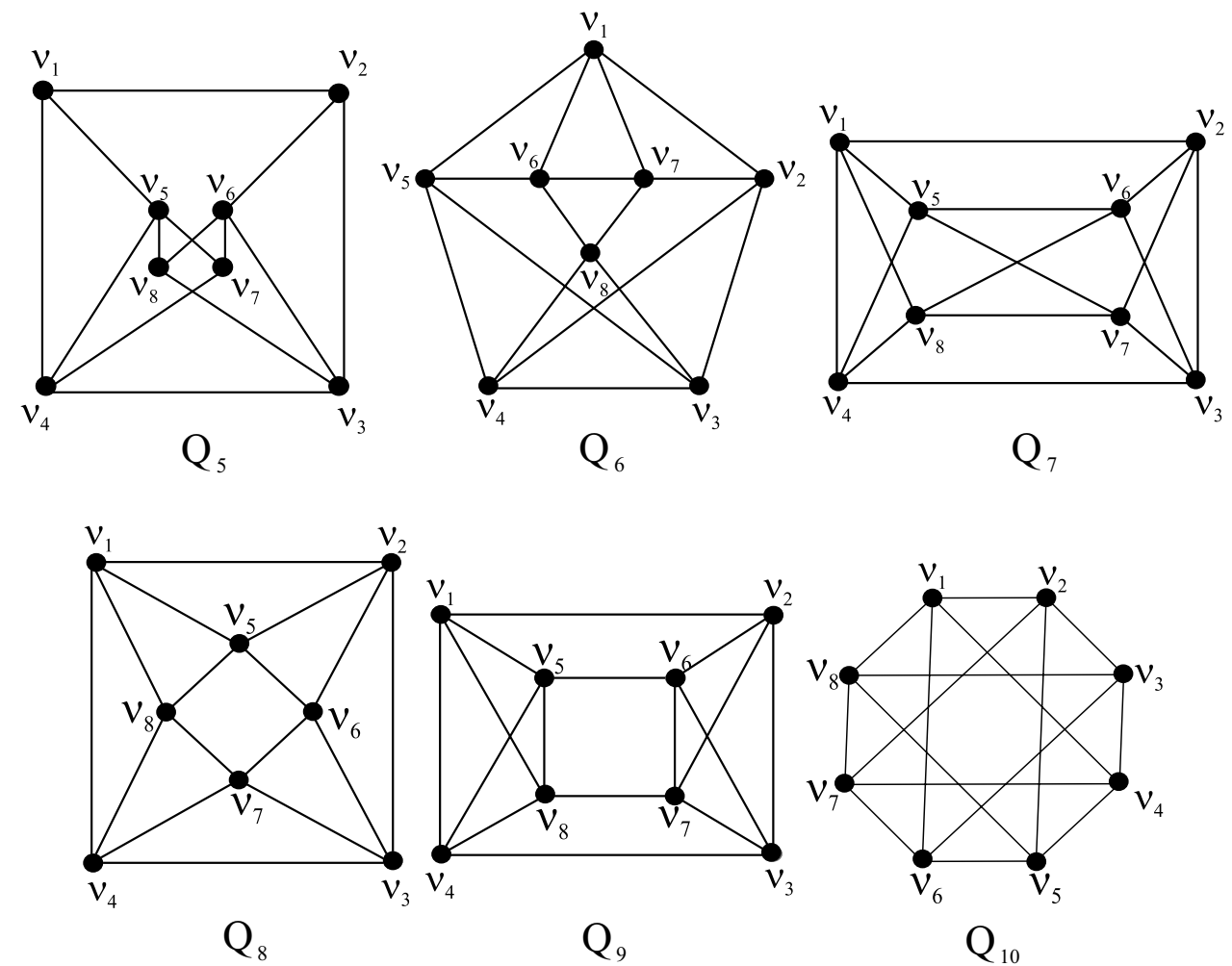

Table 1 Acceptable parameter matrices of quartic graph with order 5

Table 2 Acceptable parameter matrices of quartic graph with order 6

Table 3 Acceptable parameter matrices of quartic graph with order 7

\begin{tabular}{lllllllllll}
\hline Matrices & $M_{1}$ & $M_{2}$ & $M_{3}$ & $M_{4}$ & $M_{5}$ & $M_{6}$ & $M_{7}$ & $M_{8}$ & $M_{9}$ & $M_{10}$ \\
\hline$|B|$ & $5 / 2$ & $15 / 7$ & $5 / 3$ & 1 & $5 / 2$ & 2 & $5 / 4$ & $5 / 2$ & $5 / 3$ & $5 / 2$ \\
Acceptability & $\times$ & $\times$ & $\times$ & $\sqrt{ }$ & $\times$ & $\sqrt{ }$ & $\times$ & $\times$ & $\times$ & $\times$
\end{tabular}

\begin{tabular}{lllllllllll}
\hline Matrices & $M_{1}$ & $M_{2}$ & $M_{3}$ & $M_{4}$ & $M_{5}$ & $M_{6}$ & $M_{7}$ & $M_{8}$ & $M_{9}$ & $M_{10}$ \\
\hline$|B|$ & 3 & $18 / 7$ & 2 & $6 / 5$ & 3 & $12 / 5$ & $3 / 2$ & 3 & 2 & 3 \\
Acceptability & $\sqrt{ }$ & $\times$ & $\sqrt{ }$ & $\times$ & $\sqrt{ }$ & $\times$ & $\times$ & $\sqrt{ }$ & $\sqrt{ }$ & $\sqrt{ }$ \\
\hline
\end{tabular}

\begin{tabular}{lllllllllll}
\hline Matrices & $M_{1}$ & $M_{2}$ & $M_{3}$ & $M_{4}$ & $M_{5}$ & $M_{6}$ & $M_{7}$ & $M_{8}$ & $M_{9}$ & $M_{10}$ \\
\hline$|B|$ & $7 / 2$ & 3 & $7 / 3$ & $7 / 5$ & $7 / 2$ & $14 / 5$ & $7 / 4$ & $7 / 2$ & $7 / 3$ & $7 / 2$ \\
Acceptability & $\times$ & $\sqrt{ }$ & $\times$ & $\times$ & $\times$ & $\times$ & $\times$ & $\times$ & $\times$ & $\times$ \\
\hline
\end{tabular}

$$
M_{1}=\left(\begin{array}{ll}
0 & 4 \\
4 & 0
\end{array}\right), M_{2}=\left(\begin{array}{ll}
0 & 4 \\
3 & 1
\end{array}\right), M_{3}=\left(\begin{array}{ll}
0 & 4 \\
2 & 2
\end{array}\right), M_{4}=\left(\begin{array}{ll}
0 & 4 \\
1 & 3
\end{array}\right),
$$$$
M_{5}=\left(\begin{array}{ll}
1 & 3 \\
3 & 1
\end{array}\right)
$$$$
M_{6}=\left(\begin{array}{ll}
1 & 3 \\
2 & 2
\end{array}\right), M_{7}=\left(\begin{array}{ll}
1 & 3 \\
1 & 3
\end{array}\right), M_{8}=\left(\begin{array}{ll}
2 & 2 \\
2 & 2
\end{array}\right), M_{9}=\left(\begin{array}{ll}
2 & 2 \\
1 & 3
\end{array}\right)
$$$$
M_{10}=,\left(\begin{array}{ll}
3 & 1 \\
1 & 3
\end{array}\right) \text {. }
$$

Proof By Remark 2, Theorem 1, Proposition 2 and equality of parameter matrix, proof is clear.

Note that the word acceptable in the previous lemma states that some of the listed matrices may not be a parameter matrix of a P.2.C of $X$. Therefore, we reduce them by Proposition 1 in the next lemma (Tables 1, 2, 3, 4): 
Table 4 Acceptable parameter matrices of quartic graph with order 8

\begin{tabular}{lllllllllll}
\hline Matrices & $M_{1}$ & $M_{2}$ & $M_{3}$ & $M_{4}$ & $M_{5}$ & $M_{6}$ & $M_{7}$ & $M_{8}$ & $M_{9}$ & $M_{10}$ \\
\hline$|B|$ & 4 & $24 / 7$ & $8 / 3$ & $8 / 5$ & 4 & $16 / 5$ & 2 & 4 & $8 / 3$ & 4 \\
Acceptability & $\sqrt{ }$ & $\times$ & $\times$ & $\times$ & $\sqrt{ }$ & $\times$ & $\sqrt{ }$ & $\sqrt{ }$ & $\times$ & $\sqrt{ }$ \\
\hline
\end{tabular}

Lemma 2 Let $X$ be the quartic graph with at most 8 vertices. According to order of $X$, we can reduce the listed parameter matrices in Lemma 1 as follows:

Proof Since the number of blue vertices $(|B|)$ is not an integer, there is not exit a $P .2 . C$ of $X$ with the given parameter matrix $M_{i}$.

Corollary 1 If X be a connected quartic graph with at most 8 vertices, then only the matrices listed in the following table are acceptable as parameter matrices of a P.2.C of $X$ (Table 5):

\section{Perfect 2-coloring of the quartic graphs with order at most 8}

In the previous section, we determined all acceptable parameter matrices of a P.2.C of a connected quartic graph with at most 8 vertices. In this section, we show which of them have a structure. In the other words, we introduce a perfect 2-coloring $C$ for them.

Lemma 3 Assume that $X$ be a connected k-regular graph. Then there are no P.2.C with parameter matrix $M=\left(\begin{array}{cc}a & b \\ a^{\prime} & b^{\prime}\end{array}\right)$ such that $a=|B| \leq k$.

Proof Suppose, contrary to our claim, there is a P.2.C with presumed conditions. Let $V$ be an arbitrary vertex with blue color. So, since $a=k$, we conclude that all $k$ vertices adjacent to $v$ must be blue. This is in contradiction with $|B| \leq k$.

Corollary 2 There are no P.2.C of $Q_{2}$ with the matrices $M_{9}$ and $M_{10}$.

Proof From Lemma 2, we conclude that graph $Q_{2}$ has the conditions of Lemma 3, So the proof is straightforward.
We will discuss about acceptability of matrix $M_{7}$ is the following lemma:

Lemma 4 There are no P.2.C of graphs $Q_{6}, Q_{7}$ and $Q_{8}$ with matrix $M_{7}$.

Proof Suppose that there is a P.2.C of the graphs with $M_{7}$. Note that both adjacent vertices of the graph $Q_{6}, Q_{7}$ and $Q_{8}$ have a common vertex. On the other hand, from $\alpha_{11}=1$ in $M_{7}$, we obtain that there are two adjacent vertices with blue color. So, these two blue vertices have common red vertex. This is a contradiction with $\alpha_{21}=1$.

Lemma 5 There are no P.2.C of graphs $Q_{5}, Q_{6}, Q_{7}, Q_{8}$ and $Q_{9}$ with the matrices $M_{1}$ and $M_{10}$.

Proof The proof of this lemma is similar to the proof of Lemma 4.

In the following, we will state the main result of this article. For this, we determine which class of the quartic graphs have a structure.

Theorem 2 Only parameter matrices of P.2.C of $Q_{1}$ are $M_{4}$ and $M_{6}$.

Proof By Corollary 1, only matrices $M_{4}$ and $M_{6}$ are acceptable as parameter matrices of a P.2.C of $Q_{1}$. Two mapping $C_{1}$ and $C_{2}$ determine two structures for $Q_{1}$ with matrices $M_{4}$ and $M_{6}$, respectively, as follows:

$$
C_{1}\left(v_{1}\right)=1, C_{1}\left(v_{2}\right)=C_{1}\left(v_{3}\right)=C_{1}\left(v_{4}\right)=C_{1}\left(v_{5}\right)=2 \quad,
$$
$C_{2}\left(v_{1}\right)=C_{2}\left(v_{2}\right)=1, C_{2}\left(v_{3}\right)=C_{2}\left(v_{4}\right)=C_{2}\left(v_{5}\right)=2$.

It is clear that $C_{1}$ and $C_{2}$ are P.2.C with matrices $M_{4}$ and $M_{6}$, respectively.

Theorem 3 The parameter matrices of P.2.C of $Q_{2}$ are $M_{3}$ and $M_{8}$.

Proof From Corollary 1, we deduce that acceptable parameter matrices of $Q_{2}$ are $M_{1}, M_{3}, M_{5}, M_{8}, M_{9}$ and $M_{10}$. We next show that only $M_{3}$ and $M_{8}$ have a structure. For this, let $C_{1}$ and $C_{2}$ be given by
Table 5 Acceptable parameter matrices of quartic graph with at most order 8

\begin{tabular}{lllll}
\hline Graphs & Order 5 & Order 6 & Order 7 & Order 8 \\
\hline Matrices & $M_{4}, M_{6}$ & $M_{1}, M_{3}, M_{5}, M_{8}, M_{9}, M_{10}$ & $M_{2}$ & $M_{1}, M_{5}, M_{7}, M_{8}, M_{10}$ \\
\hline
\end{tabular}


$C_{1}\left(v_{1}\right)=C_{1}\left(v_{6}\right)=1, C_{1}\left(v_{2}\right)=C_{1}\left(v_{3}\right)=C_{1}\left(v_{4}\right)=C_{1}\left(v_{5}\right)=2$,

$C_{2}\left(v_{1}\right)=C_{2}\left(v_{3}\right)=C_{2}\left(v_{4}\right)=1, C_{2}\left(v_{2}\right)=C_{2}\left(v_{5}\right)=C_{2}\left(v_{6}\right)=2$.

It is obvious that the function $C_{1}$ and $C_{2}$ stats a P.2.C for $Q_{2}$ with matrices $M_{3}$ and $M_{8}$, respectively. In the following, we prove that other matrices can not be as parameter matrices of $Q_{2}$. First, we show that there is no P.2.C with the matrix $M_{1}$ for the graph $Q_{2}$. Contrary to our claim, suppose that $C$ is a $P$.2.C of $Q_{2}$ with the matrix $M_{1}$. There is no loss of generality in assuming $C\left(v_{1}\right)=1$. From $M_{1}$, we conclude that $C\left(v_{2}\right)=C\left(v_{3}\right)=C\left(v_{4}\right)=C\left(v_{5}\right)=2$. Hence only one vertex remains, even if it is blue, in contradiction with the number of blue vertices $(|B|=3)$.

We have shown in Corollary 2 that there is no P.2.C of $Q_{2}$ with the matrices $M_{9}$ and $M_{10}$.

Now we prove that there is no P.2.C of $Q_{2}$ with the matrix $M_{5}$. Suppose that there is a P.2.C of $Q_{2}$ with the matrix $M_{5}$, say $C$. From $\alpha_{11}=1$, without loss of generality, we assume that $C\left(v_{1}\right)=C\left(v_{2}\right)=1$. Thus, from $\alpha_{12}=3$, we have $C\left(v_{3}\right)=C\left(v_{4}\right)=C\left(v_{5}\right)=C\left(v_{6}\right)=2$. In this case, the red vertex $v_{6}$ is connected to the other three red vertices, which leads to a contradiction.

Theorem 4 Only the graph $Q_{4}$ has a P.2.C between the quartic graphs with 7 vertices.

Proof According to Corollary 1, we see that only parameter matrix of $Q_{3}$ and $Q_{4}$ is $M_{2}$. First, we give a structure for $Q_{4}$ with matrix $M_{2}$ by defining the function $C$ as follows:

$C\left(v_{1}\right)=C\left(v_{2}\right)=C\left(v_{3}\right)=C\left(v_{4}\right)=2, C\left(v_{5}\right)=C\left(v_{6}\right)=C\left(v_{7}\right)$

It is easy to check that $C$ is a P.2.C of $Q_{4}$ with matrix $M_{2}$. Now we prove that there is no P.2. $C$ with the matrix $M_{2}$ for the graph $Q_{3}$. Let $C$ be a perfect 2-coloring of $Q_{3}$ with the matrix $M_{2}$. Let $C\left(v_{1}\right)=1$. From $\alpha_{12}=4$, we deduce that all vertices are red except for adjacent vertices $v_{4}$ and $v_{5}$. On the other hand, we know that $|B|=3$. Thus $C\left(v_{4}\right)=C\left(v_{5}\right)=1$. This is a contradiction with $\alpha_{11}=0$.

Finally, we get the following theorem (Table 6):

Theorem 5 The parameter matrices of a P.2.C of a quartic graph of order 8 are listed in the following table:

Proof From Corollary 1, we conclude that acceptable parameter matrices of the quartic graphs with order 8 are $M_{1}, M_{5}, M_{7}, M_{8}$ and $M_{10}$. First, we determine structures for the quartic graphs with order 8 with their listed parameter matrices in Table 6 as follows:

Structure for $Q_{5}$ with matrix $M_{7}$ :

$C\left(v_{1}\right)=C\left(v_{2}\right)=1, C\left(v_{j}\right)=2$ for $j=3,4, \ldots, 8$.

Structure for $Q_{5}$ with matrix $M_{8}$ :

$C\left(v_{1}\right)=C\left(v_{2}\right)=C\left(v_{3}\right)=C\left(v_{4}\right)=1, C\left(v_{j}\right)=2$ for $j=5,6, \ldots, 8$.

Structure for $Q_{7}$ with matrix $M_{7}$ :
Table 6 Parameter matrices of the quartic graphs of order 8

\begin{tabular}{lccccc}
\hline $\begin{array}{l}\text { Matrices } \\
\text { graphs }\end{array}$ & $M_{1}$ & $M_{5}$ & $M_{7}$ & $M_{8}$ & $M_{10}$ \\
\hline$Q_{5}$ & $\times$ & $\times$ & $\sqrt{ }$ & $\sqrt{ }$ & $\times$ \\
$Q_{6}$ & $\times$ & $\times$ & $\times$ & $\times$ & $\times$ \\
$Q_{7}$ & $\times$ & $\times$ & $\sqrt{ }$ & $\sqrt{ }$ & $\times$ \\
$Q_{8}$ & $\times$ & $\sqrt{ }$ & $\times$ & $\sqrt{ }$ & $\times$ \\
$Q_{9}$ & $\times$ & $\sqrt{ }$ & $\sqrt{ }$ & $\sqrt{ }$ & $\times$ \\
$Q_{10}$ & $\sqrt{ }$ & $\times$ & $\sqrt{ }$ & $\sqrt{ }$ & $\times$ \\
\hline
\end{tabular}

$C\left(v_{1}\right)=C\left(v_{2}\right)=1, C\left(v_{j}\right)=2$ for $j=3,4, \ldots, 8$.

Structure for $Q_{7}$ with matrix $M_{8}$ :

$C\left(v_{1}\right)=C\left(v_{2}\right)=C\left(v_{3}\right)=C\left(v_{4}\right)=1, C\left(v_{j}\right)=2$ for $j=5,6, \ldots, 8$.

Structure for $Q_{8}$ with matrix $M_{5}$ :

$C\left(v_{1}\right)=C\left(v_{3}\right)=C\left(v_{5}\right)=C\left(v_{7}\right)=1, C\left(v_{2}\right)=C\left(v_{4}\right)=C\left(v_{6}\right)$

$=C\left(v_{8}\right)=2$.

Structure for $Q_{8}$ with matrix $M_{8}$ :

$C\left(v_{1}\right)=C\left(v_{2}\right)=C\left(v_{3}\right)=C\left(v_{4}\right)=1, C\left(v_{j}\right)=2$ for $j=5,6, \ldots, 8$.

Structure for $Q_{9}$ with matrix $M_{5}$ :

$C\left(v_{1}\right)=C\left(v_{3}\right)=C\left(v_{5}\right)=C\left(v_{7}\right)=1, C\left(v_{2}\right)=C\left(v_{4}\right)$

$=C\left(v_{6}\right)=C\left(v_{8}\right)=2$.

Structure for $Q_{9}$ with matrix $M_{7}$ :

$C\left(v_{1}\right)=C\left(v_{2}\right)=1, C\left(v_{j}\right)=2$ for $j=3,4, \ldots, 8$.

Structure for $Q_{9}$ with matrix $M_{8}$ :

$C\left(v_{1}\right)=C\left(v_{2}\right)=C\left(v_{3}\right)=C\left(v_{4}\right)=1, C\left(v_{5}\right)=C\left(v_{6}\right)$

$=C\left(v_{7}\right)=C\left(v_{8}\right)=2$.

1Structure for $Q_{10}$ with matrix $M_{1}$ :

$C\left(v_{1}\right)=C\left(v_{3}\right)=C\left(v_{5}\right)=C\left(v_{7}\right)=1, C\left(v_{2}\right)=C\left(v_{4}\right)$

$=C\left(v_{6}\right)=C\left(v_{8}\right)=2$.

Structure for $Q_{10}$ with matrix $M_{7}$ :

$C\left(v_{1}\right)=C\left(v_{2}\right)=1, C\left(v_{j}\right)=2$ for $j=3,4, \ldots, 8$.

Structure for $Q_{10}$ with matrix $M_{8}$ :

$C\left(v_{1}\right)=C\left(v_{2}\right)=C\left(v_{3}\right)=C\left(v_{4}\right)=1, C\left(v_{5}\right)=C\left(v_{6}\right)$

$=C\left(v_{7}\right)=C\left(v_{8}\right)=2$.

It is easily to see that the above functions are perfect 2-coloring with their expressed parameter matrices.

Note that, from Lemma 5, we see that there is no perfect 2-coloring for $Q_{5}, Q_{6}, Q_{7}, Q_{8}$ and $Q_{9}$ with the matrix $M_{1}$.

Also, there is no perfect 2-coloring of $Q_{5}$ with the matrix $M_{5}$. On the contrary, suppose that $C$ is a perfect 2-coloring of $Q_{5}$ with the matrix $M_{5}$. There is no loss of generality in assuming $C\left(v_{1}\right)=C\left(v_{2}\right)=1$. From $\alpha_{21}=3$ and $C\left(v_{1}\right)=1$, we obtain $C\left(v_{4}\right)=C\left(v_{5}\right)=2$. On the other hand, from $\alpha_{21}=3$ and $C\left(v_{2}\right)=1$, we have $C\left(v_{7}\right)=C\left(v_{3}\right)=2$. Thus vertex $v_{4}$ with red color is adjacent to the other two red vertices $\left(v_{3}\right.$ and $v_{5}$ ), contrary to $\alpha_{22}=1$. The proof for $Q_{5}$ with matrix $M_{10}$ is similar.

Now, we show there are no P.2.C of $Q_{6}$ with any matrices $M_{1}, M_{5}, M_{7}, M_{8}$ and $M_{10}$. From Lemmas 4 and 5, we see that there is no P.2.C of $Q_{6}$ with matrices $M_{7}, M_{1}$ and $M_{10}$, respectively. It suffices to show that there is no 
Table 7 All parameter matrices of the quartic graphs up to order 8

\begin{tabular}{ll}
\hline Graphs & Parameter matrices of a perfect 2-coloring \\
\hline$Q_{1}$ & $M_{4}, M_{6}$ \\
$Q_{2}$ & $M_{3}, M_{8}$ \\
$Q_{3}$ & The $Q_{3}$ has no any perfect 2-coloring \\
$Q_{4}$ & $M_{2}$ \\
$Q_{5}$ & $M_{7}, M_{8}$ \\
$Q_{6}$ & The $Q_{6}$ has no any perfect 2-coloring \\
$Q_{7}$ & $M_{7}, M_{8}$ \\
$Q_{8}$ & $M_{5}, M_{8}$ \\
$Q_{9}$ & $M_{5}, M_{7}, M_{8}$ \\
$Q_{10}$ & $M_{1}, M_{7}, M_{8}$ \\
\hline
\end{tabular}

P.2.C of $Q_{6}$ with matrices $M_{5}$ and $M_{8}$. Suppose that $C$ is a P.2.C of $Q_{6}$ with matrix $M_{5}$. Let $C\left(v_{1}\right)=1$. From $\alpha_{11}=1$ we deduce that each of $v_{2}, v_{5}, v_{6}$ or $v_{7}$ can be the only blue vertex adjacent to $v_{1}$. Without restriction of generality, we can assume that $C\left(v_{2}\right)=1$. Thus, from $M_{5}$ we have $C\left(v_{3}\right)=C\left(v_{4}\right)=C\left(v_{5}\right)=C\left(v_{6}\right)=C\left(v_{7}\right)=2$, which is a contradiction with $\alpha_{22}=1$. The same conclusion can be drawn for $M_{8}$.

For graph $Q_{7}$, we only need to show that there is no P.2.C of $Q_{7}$ with matrix $M_{5}$. Suppose there is a P.2.C of $Q_{7}$ with matrix $M_{5}$. Without loss of generality, we can suppose that $C\left(v_{1}\right)=C\left(v_{2}\right)=1$. Since $\alpha_{12}=3$, we have $C\left(v_{6}\right)=C\left(v_{7}\right)=C\left(v_{8}\right)=2$, contrary to $\alpha_{22}=1$. It remains only to prove that there is no P.2.C of $Q_{10}$ with matrix $M_{5}$. On the contrary, suppose that there is a P.2.C of $Q_{10}$ with matrix $M_{5}$. From $\alpha_{11}=1$, we conclude that the two adjacent vertices in $Q_{10}$, all other vertices will be red. This gets to a contradiction with $|B|=4$, and the proof is complete.

Corollary 3 The only P.2.C of the quartic graphs with at most 8 vertices is prefect coloring with listed matrices in the following table: (Table 7).

\section{Conclusion}

The perfect coloring of graphs is closely related to coding theory, algebraic theory, graph theory and combinatorics, including designs. We can consider perfect $m$-coloring as a generalization of the concept of completely regular codes presented by P. Delsarte for the first time. This class of codes has been of interest to coding theorists and graph theorists alike. In this way, the problem of existence of perfect coloring of the Johnson graphs, the generalized Petersen graphs and the $n$-dimensional hypercube graphs $Q_{n}$ for $n<24$ has been settled in [2-4, 6] and [5].

In this paper, we have listed all parameters of existing perfect 2-coloring in the quartic graphs with order at most 8 . The question of existence of perfect 3-coloring of the quartic graphs remains open.

Open Access This article is distributed under the terms of the Creative Commons Attribution 4.0 International License (http://creativeco mmons.org/licenses/by/4.0/), which permits unrestricted use, distribution, and reproduction in any medium, provided you give appropriate credit to the original author(s) and the source, provide a link to the Creative Commons license, and indicate if changes were made.

\section{References}

1. Ahlswede, R., Aydinian, H.K., Khachatrian, L.H.: On perfect codes and related concepts. Des. Codes Cryptogeraphy 22, 227 237 (2001)

2. Alaeiyan, M., Karami, H.: Perfect 2-coloring of the generalized Petersen graph. Proc. Indian Acad. Sci. N.A 126, 289-294 (2016)

3. Avgustinovich, S.V., Mogilnykh, IYu.: Perfect coloring of the Johnson graph $J(8,3)$ and $J(8,4)$ with two colors. J. Appl. Ind. Math. 5, 19-30 (2011)

4. Avgustinovich, S.V., Mogilnykh, I.Yu.: Perfect 2-coloring of Johnson Graph $J(6,3)$ and $J(7,3)$. n Lecture Notes in Computer Science, vol. 5228, pp. 11-19. Springer (2008)

5. Fon-der-Flaass, D.G.: Perfect 2-colorings of a Hypercube. Sib. Math. Zh. 48(4), 923-930 (2007)

6. Gavilyuk, A.L., Goryainov, S.V.: On perfect 2-coloring of Johnson graphs $J(v, 3)$. J. Combin. Des. 21, 232-252 (2013)

7. Godsil, C.: Compact graphs and equitable partitions. Linear Slgebra Aappl. 255, 259-266 (1997)

8. Mogilnykh, IYu.: On the regularity of perfect 2-coloring of the Johnson graph. Problemy Peredachi Inform. 43(4), 37-44 (2007)

9. Ronald, C.R.: An Atlas of Graphs. University of Waterloo, Waterloo (1998)

Publisher's Note Springer Nature remains neutral with regard to jurisdictional claims in published maps and institutional affiliations. 